T. Suzuki

Nagoya Math. J.

Vol. 77 (1980), 145-166

\title{
WEIL TYPE REPRESENTATIONS AND AUTOMORPHIC FORMS
}

\author{
TOSHIAKI SUZUKI
}

\section{Introduction}

During 1934-1936, W. L. Ferrar investigated the relation between summation formulae and Dirichlet series with functional equations, inspired by Voronoi's works (1904) on summation formula with respect to the numbers of divisors. In [11] A. Weil showed that the automorphic properties of theta series are expressed by generalized Poisson summation formulae with respect to the so-called Weil representation. On the other hand, T. Kubota, in his study of the reciprocity law in a number field, defined a generalized theta series and a generalized Weil type representation of $S L(2, C)$ and obtained similar results to those of A. Weil (1970 1976, [5], [6], [7]). The methods, used by W. L. Ferrar and T. Kubota, to obtain (generalized Poisson) summation formulae depend similarly on functional equations of Dirichlet series (attached to the generalized theta series).

In this paper, we will show the relation between automorphic forms and generalized Poisson summation formulae with respect to "Weil type representations" by a simple and direct way. In $\S 1$, we define "Weil type representations" $r_{s, k}$ of $S L(2, R)$ and "Whittaker functions" related to $r_{s, k}$. A "whittaker function" has a intertwining property with respect to modular transformations and "Weil type representations". This corresponds to the fact that the function $\exp \left(-\frac{x^{2}}{2}\right)$ is invariant under Fourier transformation. In $\S 2$, we show that Eisenstein series of $S L(2, R)$ can be expanded by "Whittaker functions". The generalized Poisson summation formula for Eisenstein series is proved by this fact together with the intertwining property. In $\S 3$, we see that the generalized Poisson summation formulae for automorphic forms are equivalent to their automorphic properties. In $\S 4$, we consider "Weil type representations" for

Received February 13, 1979. 
covering groups of $S L(2, R)$ and "Whittaker functions" related to them. Then we obtain the original Weil representation as a special case.

The author wishes to express his hearty thanks to Professor T. Kubota, for his valuable advice and warm encouragement.

\section{Notation}

We denote by $N, Z, R$ and $C$ the set of natural numbers, the ring of rational integers, the real number field and the complex number field, respectively. For $z \in C$, we put $e(z)=\exp (2 \pi \sqrt{-1} z)$. We denote by $\bar{z}$ the complex conjugate of $z \in C$. For $x \in R$, we put

$$
\operatorname{sgn}(x)= \begin{cases}1 & \text { if } x>0 \\ -1 & \text { if } x<0 \\ 0 & \text { if } x=0\end{cases}
$$

\section{§1. Representations of $S L(2, R)$}

1. To begin with, we consider the representations $\pi_{s, k}$ of $S L(2, R)$ which are fully investigated by I. M. Gel'fand, M. I. Graev and N. Ya. Vielenkin ([2], chap. VII).

For any integer $k$ and any complex number $s$, we associate a function space $\mathscr{A}_{s, k}$ consisting of the function $\phi(x), x \in R$, with the following properties.

(1) Every $\phi(x)$ must possess all derivatives.

(2) Asymptotically as $|x| \rightarrow \infty$, the behavior of $\phi(x)$ is given by

$$
\phi(x) \sim C|x|^{-2 s+k} x^{-k}
$$

where $C$ is a constant.

We topologize $\mathscr{A}_{s, k}$ in the following way. A sequence of functions $\phi_{n}$ in $\mathscr{A}_{s, k}$ is said to "converge to zero" if the $\phi_{n}$ converge uniformly to zero with all their derivatives on every compact set of $\boldsymbol{R}$.

The representation $\pi_{s, k}$ of $S L(2, R)$ in the space $\mathscr{A}_{s, k}$ is defined as follows:

$$
\pi_{s, k}(\sigma) \phi(x)=\phi\left(\frac{\alpha x+\beta}{\gamma x+\delta}\right)|\gamma x+\delta|^{-2 s} \operatorname{sgn}^{k}(\gamma x+\delta),
$$

where $\sigma=\left(\begin{array}{ll}\alpha & \beta \\ \gamma & \delta\end{array}\right) \in S L(2, \boldsymbol{R})$ and $\phi(x) \in \mathscr{A}_{s, k}$. 
We shall summarize the results on the representations $\pi_{s, k}$ of $S L(2, R)$ according to [2].

If $2 s \not \equiv k(\bmod 2)$, then $\pi_{s, k}$ is irreducible. Two representations $\pi_{s, k}$ and $\pi_{s^{\prime}, k^{\prime}}\left(2 s \neq k(\bmod 2), 2 s^{\prime} \neq k^{\prime}(\bmod 2)\right)$ are equivalent if and only if $k \equiv k^{\prime}(\bmod 2), s=s^{\prime}$ or $k \equiv k^{\prime}(\bmod 2), s=1-s^{\prime}$. There exists an intertwining operator $M_{s, k}$ from $\mathscr{A}_{s, k}$ onto $\mathscr{A}_{1-s, k}$, i.e.,

$$
M_{s, k} \pi_{s, k}(\sigma)=\pi_{1-s, k}(\sigma) M_{s, k}, \quad \sigma \in S L(2, R) .
$$

Here, $M_{s, k}$ is given by

$$
M_{s, k} \phi(x)=\int_{-\infty}^{\infty}\left|x_{1}-x\right|^{2 s-2} \operatorname{sgn}^{k}\left(x_{1}-x\right) \phi\left(x_{1}\right) d x_{1}, \quad \phi(x) \in \mathscr{A}_{s, k},
$$

where the integral is understood in the sense of its regularization (for details, see [2]).

If $2 s \equiv k(\bmod 2)$, then $\pi_{s, k}$ is not irreducible. To see this, it is sufficient to consider only $\pi_{0, k}, k \in Z$. We write $\pi_{k}$ (resp. $\mathscr{A}_{k}$ ) instead of $\pi_{0, k}$ (resp. $\left.\mathscr{A}_{0, k}\right)$. First, consider the case of nonpositive integer $k=1-n(n \in N)$. Obviously, $\mathscr{A}_{1-n}$ contains the invariant subspace $E_{1-n}$ of dimension $n$, consisting of polynomials of degree $n-1$ and lower. There exist also two other invariant subspaces $\mathscr{A}_{1-n}^{+}$and $\mathscr{A}_{1-n}^{-}$in $\mathscr{A}_{1-n}$. $\mathscr{A}_{1-n}^{+}$(resp. $\mathscr{A}_{1-n}^{-}$) consists of functions which are the boundary values of holomorphic functions in the upper (resp. lower) half plane up to polynomials of degree $n-1$ and lower. Further, $\mathscr{A}_{1-n}^{+} \cap \mathscr{A}_{1-n}^{-}=E_{1-n}, \mathscr{A}_{1-n}^{+} \cup \mathscr{A}_{1-n}^{-}=\mathscr{A}_{1-n}$. Next, consider the case of integer $k$ such that $k=n+1$ for $n \in N$. $\mathscr{A}_{n+1}$ contains the subspace $F_{n+1}$, which consists of functions $f(x)$ with

$$
\int_{-\infty}^{\infty} x^{l} f(x) d x=0
$$

for $l=0,1, \cdots, n-1$. Obviously, the quotient space $\mathscr{A}_{n+1} / F_{n+1}$ is of dimension $n$. Further, $F_{n+1}$ has two other invariant subspaces $F_{n+1}^{+}$and $F_{n+1}^{-}$. $\quad F_{n+1}^{+}$(resp. $F_{n+1}^{-}$) consists of functions which are the boundary values of holomorphic functions in the upper (resp. lower) half plane. There exists an intertwining operator $M_{1-n}$ from $\mathscr{A}_{1-n}$ onto $F_{n+1}$ whose kernel is $E_{1-n}$. $\quad M_{1-n}$ is given by

$$
M_{1-n} \phi(x)=\frac{d^{n}}{d x^{n}} \phi(x)=\phi^{(n)}(x)
$$

More precisely, there exists an intertwining operator $M_{1-n}^{+}$(resp. $M_{1-n}^{-}$) 
from $\mathscr{A}_{1-n}^{+}$(resp. $\mathscr{A}_{1-n}^{-}$) onto $F_{n+1}^{+}\left(\right.$resp. $\left.F_{n+1}^{-}\right)$whose kernel is $E_{1-n}$. $M_{1-n}^{ \pm}$ are given by

$$
M_{1-n}^{ \pm} \phi(x)=\frac{1}{2 \pi \sqrt{-1}} \int_{-\infty}^{\infty} \frac{\phi(x)}{\left(x_{1}-x \mp \sqrt{-1} 0\right)^{n+1}} d x
$$

where $(x-\sqrt{-1} 0)^{-n}$ (resp. $\left.(x+\sqrt{-1} 0)^{-n}\right)$ is the boundary value of $(x-\sqrt{-1} y)^{-n}$ (resp. $(x+\sqrt{-1} y)^{-n}$ ) when $y \rightarrow 0$ (for details, see [2]). Further, there exists an intertwining operator $M_{n+1}$ from $\mathscr{A}_{n+1}$ onto $E_{1-n}$ whose kernel is $F_{n+1} \cdot M_{n+1}$ is given by

$$
M_{n+1} \phi(x)=\int_{-\infty}^{\infty}\left(x_{1}-x\right)^{n-1} \phi\left(x_{1}\right) d x_{1} .
$$

Similarly, we have $\mathscr{A}_{1}=F_{1}^{+} \oplus F_{1}^{-}$where $F_{1}^{+}$(resp. $F_{1}^{-}$) consists of functions which are boundary values of holomorphic functions in the upper (resp. lower) half plane.

The representation $\pi_{s, k}$ becomes a unitary representation if and only if $\operatorname{Re}(s)=\frac{1}{2}$ or $0<s<1\left(s \neq \frac{1}{2}\right), k \equiv 0(\bmod 2)$. When $\operatorname{Re}(s)=\frac{1}{2}$, this is called a representation of the principal series and the scalar product in $\mathscr{A}_{s, k}$ is of the form

$$
(\phi, \psi)_{s, k}=\int_{-\infty}^{\infty} \phi(x) \bar{\psi}(x) d x, \quad \phi, \psi \in \mathscr{A}_{s, k} .
$$

When $0<s<1\left(s \neq \frac{1}{2}\right), k \equiv 0(\bmod 2)$, the unitary representation $\pi_{s, k}$ is called a representation of the supplementary series and the scalar product in $\mathscr{A}_{s, k}$ is of the form

$$
(\phi, \psi)_{s, k}=\int_{-\infty}^{\infty} \int_{-\infty}^{\infty}\left|x_{1}-x\right|^{2 s-2} \phi\left(x_{1}\right) \bar{\psi}(x) d x_{1} d x, \quad \phi, \psi \in \mathscr{A}_{s, k} .
$$

The representations $\left(\pi_{1}, F_{1}\right)$ and $\left(\pi_{n+1}, F_{n+1}\right) \cong\left(\pi_{1-n}, \mathscr{A}_{1-n} / E_{1-n}\right)$ are also unitary representations, which are called representations of the discrete series. The scalar products in $F_{1}$ and $F_{n+1}$ are given as follows:

$$
\begin{gathered}
(\phi, \psi)_{1}^{ \pm}=\int_{-\infty}^{\infty} \phi(x) \bar{\psi}(x) d x, \quad \phi, \psi \in F_{1}^{ \pm}, \\
(\phi, \psi)_{n+1}^{ \pm}=\int_{-\infty}^{\infty} \int_{-\infty}^{\infty}\left(x_{1}-x\right)^{n-1} \ln \left(x_{1}-x \mp \sqrt{-1} \cdot 0\right) \phi\left(x_{1}\right) \bar{\psi}(x) d x_{1} d x, \\
\phi, \psi \in F_{n+1}^{ \pm} .
\end{gathered}
$$

Here, $\ln (x \pm \sqrt{-1} \cdot 0)$ are the boundary values of $\ln (x \pm \sqrt{-1} \cdot y)$ 
$=\log (x \pm \sqrt{-1} \cdot y)$ when $y \rightarrow 0(y>0)$ (for details, see [2]).

2. We denote by $H$ the complex upper half plane $\{z=\xi+\sqrt{-1} \cdot \eta$ $\in C: \eta>0\}$. An element $\sigma=\left(\begin{array}{ll}\alpha & \beta \\ \gamma & \delta\end{array}\right) \in S L(2, R)$ operates on $H$ (and on its boundary $R \cup\{\infty\}$ ) by means of the fractional linear transformation

$$
z \mapsto \sigma z=\frac{\alpha z+\beta}{\gamma z+\delta}, \quad z \in H(z \in R \cup\{\infty\}) .
$$

Let $C_{0}(H)$ be the function space consisting of locally integrable functions with respect to the invariant measure $d z=\frac{d \xi d \eta}{\eta^{2}}$, whose support is compact.

In the following, let $k$ denote any nonnegative integer and $s$ any complex number. Set

$$
\phi_{s, k}(z, x)=\eta^{s-(k / 2)}|z-x|^{-2 s+k}(z-x)^{-k}, \quad x \in R, z=\xi+\sqrt{-1} \cdot \eta \in H,
$$

then $\phi_{s, k}(z, x) \in \mathscr{A}_{s, k}$. Especially, $\phi_{k / 2, k}(z, x) \in F_{\bar{k}}^{-}(k>0)$, because $\phi_{k / 2, k}(z, x)$ is the boundary value of $(z-x+\sqrt{-1} \cdot y)^{-k}$ as $y \rightarrow 0$. It is easy to get the following formula:

$$
\pi_{s, k}(\sigma) \phi_{s, k}(z, x)=j_{k}\left(\sigma^{-1}, z\right) \phi_{s, k}\left(\sigma^{-1} z, x\right)
$$

for any $\sigma \in S L(2, R)$. Here, $j_{k}(\sigma, z)=(\gamma z+\delta)^{-k}$ for $\sigma=\left(\begin{array}{ll}\alpha & \beta \\ \gamma & \delta\end{array}\right) \in S L(2, R)$.

For any $f \in C_{0}(H)$, set

$$
\phi_{s, k}(f, x)=\int_{H} f(z) \phi_{s, k}(z, x) d z,
$$

then we have $\phi_{s, k}(f, x) \in \mathscr{A}_{s, k}\left(\operatorname{resp} . \phi_{k / 2, k}(f, x) \in F_{k}^{-}\right)$and

$$
\pi_{s, k}(\sigma) \phi_{s, k}(f, x)=\phi_{s, k}\left(f^{\sigma, k}, x\right), \quad \sigma \in S L(2, R),
$$

where $f^{\sigma, k}(z)=f(\sigma z) j_{k}(\sigma, z)$.

If $s \neq k / 2, k \neq 0$ or $k=0,1-s \notin N$, then the set $\left\{\phi_{s, k}(f, x): f \in C_{0}(H)\right\}$ is dense in $\mathscr{A}_{s, k}$. If $s=k / 2, k \neq 0$, then the set $\left\{\phi_{s, k}(f, x): f \in C_{0}(H)\right\}$ is dense in $F_{k}^{-}$. If $k=0,1-s \in N$, then $\left\{\phi_{s, k}(f, x)\right\}=E_{2 s}$.

3. Now we try to get another realization $\left(r_{s, k}, \mathscr{B}_{s, k}\right)$ of the representation $\left(\pi_{s, k}, \mathscr{A}_{s, k}\right)$ by passing from the functions $\phi(x) \in \mathscr{A}_{s, k}$ to their Fourier transforms 


$$
\Phi(u)=\int_{-\infty}^{\infty} \phi(x) e(-u x) d x, \quad u \in \boldsymbol{R}
$$

In this realization, the representation space $\mathscr{B}_{s, k}$ consists of the Fourier transforms of $\phi(x) \in \mathscr{A}_{s, k}$, so $\mathscr{B}_{s, k}$ may contain "functions with singularities" in general.

We give the space $\mathscr{B}_{s, k}$ the topology which is induced by that of $\mathscr{A}_{s, k}$.

Denote by $\tilde{F}_{n}$ (resp. $\tilde{F}_{n}^{+}, \tilde{F}_{n}^{-}$) the function space of the Fourier transforms of $\phi(x) \in F_{n}$ (resp. $\left.F_{n}^{+}, F_{n}^{-}\right)(n \geqq 1)$. If $\Phi(u) \in \tilde{F}_{n+1}$, then

$$
\Phi^{(l)}(0)=0, \quad l=0,1, \cdots, n-1, \quad(n \in N) .
$$

If $\Phi(u) \in \tilde{F}_{n}^{+}$(resp. $\tilde{F}_{n}^{-}$), then $\Phi(u)=0$ when $u>0$ (resp. $u<0$ ).

Let $B_{s, k}(z, x), z=\xi+\sqrt{-1} \cdot \eta \in H, u \in R$, be the Fourier transform of $\phi_{s, k}(z, x)$ with respect to $x$, i.e.,

$$
\begin{aligned}
B_{s, k}(z, x) & =\int_{-\infty}^{\infty} \phi_{s, k}(z, x) e(-u x) d x \\
& =\int_{-\infty}^{\infty} \eta^{s-(k / 2)}|z-x|^{-2 s+k}(z-x)^{-k} e(-u x) d x
\end{aligned}
$$

Here, for $s$ such that $\operatorname{Re}(s)>1$, the integral converges absolutely, and for $s$ such that $\operatorname{Re}(s)<1$, the integral has a meaning as the analytic continuation on $s$. This integral can be expressed by Whittaker function $W_{\kappa, \mu}(z)$ :

$$
\begin{aligned}
& B_{s, k}(z, x) \\
& = \begin{cases}4^{1-s}(\sqrt{-1})^{-k} \frac{\Gamma(2 s-1)}{\Gamma\left(s+\frac{k}{2}\right) \Gamma\left(s-\frac{k}{2}\right)} \eta^{1-s-(k / 2)}, & \text { if } u=0, \\
\frac{\pi^{s}(\sqrt{-1})^{-k}}{\Gamma\left(s-\frac{k}{2}\right)}|u|^{s-1} \eta^{-k / 2} W_{-k / 2, s-(1 / 2)}(4 \pi|u| \eta) e(-u \xi), & \text { if } u>0, \\
\frac{\pi^{s}(\sqrt{-1})^{-k}}{\Gamma\left(s+\frac{k}{2}\right)}|u|^{s-1} \eta^{-k / 2} W_{k / 2, s-(1 / 2)}(4 \pi|u| \eta) e(-u \xi), & \text { if } u<0,\end{cases}
\end{aligned}
$$

(for the definition of $W_{x, \mu}(z)$, see [1] and [2]).

We call $B_{s, k}(z, u)$ "Whittaker function" attached to $r_{s, k}$.

For any $f \in C_{0}(H)$, set 


$$
B_{s, k}(f, u)=\int f(z) B_{s, k}(z, u) d z
$$

then $B_{s, k}(f, u)$ is the Fourier transform of $\phi_{s, k}(f, x)$. If $s \neq k / 2, k \neq 0$, or $k=0,1-s \notin N$, then the function space $\mathscr{B}_{s, k}^{0}=\left\{B_{s, k}(f, u): f \in C_{0}(H)\right\}$ is dense in $\mathscr{B}_{s, k}$. If $s=k / 2, k=0$, then the function space $\mathscr{B}_{k}^{0}=\left\{B_{s, k}(f, u)\right.$ : $\left.f \in C_{0}(H)\right\}$ is dense in $F_{k}^{-}$.

Under the assumption that $0<\operatorname{Re}(s)<1$, the formula for the operator $r_{s, k}(\sigma), \sigma=\left(\begin{array}{ll}\alpha & \beta \\ \gamma & \delta\end{array}\right) \in S L(2, R)$, takes the following form:

$$
\left(r_{s, k}(\sigma) \Phi\right)(u)=\int_{-\infty}^{\infty} K_{s, k}(\sigma: u, v) \Phi(v) d v, \quad \Phi(u) \in \mathscr{B}_{s, k},
$$

where

$$
K_{s, k}(\sigma: u, v)=\int_{-\infty}^{\infty} e\left(-u x+v \frac{\alpha x+\beta}{\gamma x+\delta}\right)|\gamma x+\delta|^{-2 s} \operatorname{sgn}^{k}(\gamma x+\delta) d x
$$

This expression can be written in a somewhat different form.

$$
\text { If } \sigma=\left(\begin{array}{ll}
\alpha & \beta \\
\gamma & \delta
\end{array}\right) \in S L(2, R), \gamma=0 \text {, then }
$$

$$
\left(r_{s, k}(\sigma) \Phi\right)(u)=\operatorname{sgn}^{k} \delta \cdot|\delta|^{-2 s+2} e(\delta \beta u) \Phi\left(\delta^{2} u\right), \quad \Phi(u) \in \mathscr{B}_{s, k}
$$

If $\sigma=\left(\begin{array}{ll}\alpha & \beta \\ \gamma & \delta\end{array}\right) \in S L(2, R), \gamma \neq 0$, then the integral kernel $K_{s, k}(\sigma: u, v)$ can be written in

$$
K_{s, k}(\sigma: u, v)=|\gamma|^{-1} e\left(\frac{\delta u+\alpha v}{\gamma}\right) \int_{-\infty}^{\infty} e\left(-\frac{1}{\gamma}\left(u t+v t^{-1}\right)\right)|t|^{-2 s} \operatorname{sgn}^{k} t d t
$$

In the last formula, the integral converges uniformly in the region $0<\operatorname{Re}(s)<1$, and is expressed by Bessel functions. Therefore, we have: if $u v>0$, then

$$
\begin{aligned}
& K_{s, k}(\sigma: u, v)=\pi \sqrt{-1} \operatorname{sgn}^{k}(v \gamma)|\gamma|^{-1} e\left(\frac{\delta u+\alpha v}{\gamma}\right)\left|\frac{u}{v}\right|^{s-(1 / 2)} \\
& \quad \times\left[(-1)^{k} e\left(\frac{2 s-1}{4}\right) \cdot H_{2 s-1}^{(1)}\left(4 \pi \frac{\sqrt{u v}}{|\gamma|}\right)-e\left(\frac{1-2 s}{4}\right) H_{2 s-1}^{(2)}\left(4 \pi \frac{\sqrt{u v}}{|\gamma|}\right)\right],
\end{aligned}
$$

and if $u v<0$, then 


$$
\begin{aligned}
K_{s, k}(\sigma: u, v)= & 2 \cdot \operatorname{sgn}^{k}(\gamma v)|\gamma|^{-1} e\left(\frac{\delta u+\alpha v}{\gamma}\right)\left|\frac{u}{v}\right|^{s-(1 / 2)} \\
& \cdot\left[(-1)^{k} e\left(\frac{2 s-1}{4}\right)+e\left(\frac{1-2 s}{4}\right)\right] \cdot K_{2 s-1}\left(4 \pi \frac{\sqrt{|u v|}}{\gamma}\right) .
\end{aligned}
$$

Here, we quote the integral expressions of $H_{\nu}^{(1)}, H_{\nu}^{(2)}$ and $K_{\nu}$ :

$$
\begin{aligned}
& H_{\nu}^{(1)}(x)=\frac{e(-\nu / 4)}{\pi \sqrt{-1}} \int_{-\infty}^{\infty} \exp (\sqrt{-1} \cdot x \cdot \cosh (t)-\nu t) d t, \\
& (-1<\operatorname{Re}(\nu)<1, x>0) \\
& H_{\nu}^{(2)}(x)=-\frac{e(-\nu / 4)}{\pi \sqrt{-1}} \int_{-\infty}^{\infty} \exp (-\sqrt{-1} \cdot x \cdot \cosh (t)-\nu t) d t, \\
& (-1<\operatorname{Re}(\nu)<1, x>0)
\end{aligned}
$$

and

$$
\begin{aligned}
K_{\nu}(x)=\frac{1}{2} e(-\nu / 4) \int_{0}^{\infty} v^{-\nu-1} \exp & \left(-\frac{1}{2} \sqrt{-1} \cdot x\left(v-v^{-1}\right)\right) d v, \\
& (-1<\operatorname{Re}(\nu)<1, x>0),
\end{aligned}
$$

(see [10]).

The integral kernel $K_{s, k}(\sigma: u, v), \sigma=\left(\begin{array}{ll}\alpha & \beta \\ \gamma & \delta\end{array}\right) \in S L(2, R)(\gamma \neq 0)$, has a meaning for every complex number $s$, because Bessel functions are analytically continued to entire functions with respect to $s$. Taking into account of this fact, we can define the operator $r_{s, k}(\sigma), \sigma \in S L(2, R)$ in any case (i.e., for any $s$ and $k$ ) by the formula (1.7), (1.8) and (1.8)'.

According to (1.4) and to the definitions of the operator $r_{s, k}(\sigma)$ and "Whittaker function" $B_{s, k}(z, u)$, it follows that, if $0<\operatorname{Re}(s)<1$, then

$$
r_{s, k}(\sigma) B_{s, k}(z, u)=B_{s, k}\left(\sigma^{-1} z, u\right) j_{k}\left(\sigma^{-1}, z\right)
$$

for any $\sigma \in S L(2, R)$. In view of the analytic continuations on $s$, the above intertwining formula (1.9) holds for every complex number $s$. We call $\left(r_{s, k}, \mathscr{B}_{s, k}\right)$ "Weil type representation" of $S L(2, R)$.

If the representation $\left(\pi_{s, k}, \mathscr{A}_{s, k}\right)$ is unitary (in other words, "Weil type representation $\left(r_{s, k}, \mathscr{B}_{s, k}\right)$ is unitary), the scalar product in $\mathscr{B}_{s, k}$ is defined (up to a constant multiple) by

$$
(\Phi, \Psi)_{s, k}=(\phi, \psi)_{s, k}, \quad\left(\Phi, \Psi \in \mathscr{B}_{s, k}, \phi, \psi \in \mathscr{A}_{s, k}\right),
$$

where $\Phi$ and $\Psi$ are the Fourier transforms of $\phi$ and $\psi$, respectively. In 
the case of principal series:

$$
(\Phi, \Psi)_{s, k}=\int_{-\infty}^{\infty} \Phi(u) \bar{\Psi}(u) d u
$$

In the case of supplementary series:

$$
(\Phi, \Psi)_{s, k}=\int_{-\infty}^{\infty}|u|^{1-2 s} \Phi(u) \bar{\Psi}(u) d u
$$

Similarly, in the case of discrete series, the scalar products in $\tilde{F}_{n+1}^{+}$and $\tilde{F}_{n+1}^{-}(n \in N)$ are of the forms:

$$
(\Phi, \Psi)_{n+1}^{+}=(\sqrt{-1})^{-n+1} \int_{0}^{\infty} \Phi^{(n-1)}(u) \bar{\Psi}(u) d u, \quad \Phi, \Psi \in \tilde{F}_{n+1}^{+}
$$

and

$$
(\Phi, \Psi)_{n+1}^{-}=(\sqrt{-1})^{-n+1} \int_{-\infty}^{0} \Phi^{(n-1)}(u) \bar{\Psi}(u) d u, \quad \Phi, \Psi \in \tilde{F}_{n+1}^{-}
$$

respectively.

Thus, we obtain

TheOREM 1. (1) For "Weil type representation" $\left(r_{s, k}, \mathscr{B}_{s, k}\right)$ of $S L(2, R)$, defined by the formulae (1.6) and (1.7), and for "Whittaker function" $B_{s, k}(z, u)$ related to $r_{s, k}$, defined by (1.5), we have the following intertwining formula with respect to modular transformation on $z$ :

$$
r_{s, k}(\sigma) B_{s, k}(z, u)=B_{s, k}\left(\sigma^{-1} z, u\right) j_{k}\left(\sigma^{-1}, z\right), \quad \sigma \in S L(2, R) .
$$

(2) "Weil type representation" $\left(r_{s, k}, \mathscr{B}_{s, k}\right)$ is unitary if and only if $\operatorname{Re}(s)=1 / 2$ or $0<s<1(s \neq 1 / 2), k \equiv 0(\bmod 2)$, and the scalar products in these cases are given by (1.10) and (1.11), respectively.

The representations $\left(r_{(n+1) / 2, n+1}, \tilde{F}_{n+1}^{ \pm}\right)$are unitary and irreducible, and the scalar products are given by (1.12) and $(1.12)^{\prime}$, respectively.

If $2 s \neq k(\bmod 2)$, then $\left(r_{s, k}, \mathscr{B}_{s, k}\right)$ is irreducible. Two representations $r_{s, k}$ and $r_{s^{\prime}, k^{\prime}}\left(2 s \neq k(\bmod 2), 2 s^{\prime} \neq k^{\prime}(\bmod 2)\right)$ are equivalent if and only if $k \equiv k^{\prime}(\bmod 2), s=s^{\prime}$ or $k \equiv k^{\prime}(\bmod 2), s=1-s^{\prime}$.

Remark. In the cases of principal and supplementary series, the realizations $\left(r_{s, k}, \mathscr{B}_{s, k}\right)$ are already investigated ([3]). 


\section{§2. Generalized Poisson summation formula for Eisenstein series}

In this section, we write $\left(r_{s}, \mathscr{B}_{s}\right)$ and $B_{s}(z, u)$ instead of $\left(r_{s, 0}, \mathscr{B}_{s, 0}\right)$ and $B_{s, 0}(z, u)$, respectively.

First, we summarize the theory of Eisenstein series of $S L(2, R)$ (see [4]). A discontinuous subgroup $\Gamma$ of $S L(2, R)$ is called a discontinuous group of finite type, if the measure of $\Gamma \backslash H$ with respect to $d z$ is finite. Let $\kappa$ be a real number or $\infty$, and let $\Gamma_{\kappa}$ be the stabilizer of $\kappa$ in $\Gamma$, i.e. $\Gamma_{\kappa}=\{\sigma \in \Gamma \mid \sigma \kappa=\kappa\}$. We call $\kappa$ a cusp of $\Gamma$, if $\Gamma_{\kappa}$ is generated by a parabolic element. Two cusps $\kappa, \kappa^{\prime}$ are called equivalent, if there is a $\sigma \in \Gamma$ such that $\sigma \kappa=\kappa^{\prime}$. If $\Gamma$ is of finite type, then the number of inequivalent cusps is finite.

Let $\Gamma$ be a discontinuous group of finite type, and let $\kappa_{1}, \cdots, \kappa_{h}$ be a complete set of inequivalent cusps. Let $\Gamma_{i}$ be the stabilizer in $\Gamma$ of $\kappa_{i}$, i.e., $\Gamma_{i}=\left\{\sigma \in \Gamma \mid \sigma \kappa_{i}=\kappa_{i}\right\}$, and fix an element $\sigma_{i} \in S L(2, R)$ such that $\sigma_{i} \infty=\kappa_{i}$, and that $\sigma_{i}^{-1} \Gamma_{i} \sigma_{i}$ is equal to the group $\left\{\left(\begin{array}{ll}1 & \beta \\ 0 & 1\end{array}\right) \mid \beta \in Z\right\}$. Furthermore, denote by $\eta(z)$ the imaginary part of $z \in H$.

The Eisenstein series $E_{i}(z, x)$ for the cusp $\kappa_{i}$, is defined by

$$
E_{i}(z, s)=\sum_{\sigma} \eta\left(\sigma_{i}^{-1} \sigma z\right)^{s}, \quad\left(\sigma \in \Gamma_{i} \backslash \Gamma\right),
$$

where $s$ is a complex variable.

It is known that the Eisenstein series $E_{i}(z, s)$ converges absolutely, if $\operatorname{Re}(s)>1$. From the definition, it follows immediately that $E_{i}(\sigma z, s)$ $=E_{i}(z, s)$ for any $\sigma \in \Gamma$, and $D E_{i}(z, s)=\lambda E_{i}(z, s)$ with $\lambda=s(s-1)$, where $D$ is the Laplacian on $H$ given by

$$
D=\eta^{2}\left(\frac{\partial^{2}}{\partial \xi^{2}}+\frac{\partial^{2}}{\partial \eta^{2}}\right), \quad z=\xi+\sqrt{-1} \cdot \eta .
$$

Further, $E_{i}(z, s)$ does not depend on the particular choice of $\sigma_{i}$ and a cusp $\kappa_{i}$ among equivalent ones.

In the following, we assume that $\infty$ is a cusp and the stabilizer $\Gamma_{\infty}=\{\sigma \in \Gamma: \sigma \infty=\infty\}$ of $\infty$ is equal to the group $\left\{\left(\begin{array}{ll}1 & \beta \\ 0 & 1\end{array}\right) \mid \beta \in Z\right\}$.

A function $f(z)$ on $H$ is called an automorphic function with respect to $\Gamma$, if $f(\sigma z)=f(z)$ holds for all $\sigma \in \Gamma$. If $\kappa$ is a cusp of $\Gamma$, then there exist a $\sigma^{\prime} \in S L(2, R)$ such that $\sigma^{\prime} \infty=\kappa$. If $f(z)$ is an automorphic function, then $f\left(\sigma^{\prime} z\right)$ is a periodic function with period 1, i.e., $f\left(\sigma^{\prime}(z+1)\right)$ 
$=f\left(\sigma^{\prime} z\right)$. Therefore, we have a Fourier series expansion

$$
f\left(\sigma^{\prime} z\right)=\sum_{m} a_{m}(\eta) e(m \xi), \quad(m \in Z, z=\xi+\sqrt{-1} \cdot \eta),
$$

whenever $f$ satisfies some natural conditions. Here,

$$
a_{m}(\eta)=\int_{0}^{1} f\left(\sigma^{\prime} z\right) e(-m \xi) d \xi .
$$

The Fourier expansion of the Eisenstein series $E_{i}(z, s)$ at the cusp $\kappa_{j}$, is given in the form

$$
E_{i}\left(\sigma_{j} z, s\right)=\sum_{m} a_{i j, m}(\eta, s) e(-m \xi), \quad(m \in Z),
$$

with

$$
\begin{aligned}
a_{i j, m}(\eta, s) & =\int_{0}^{1} E_{i}\left(\sigma_{j} z, s\right) e(m \xi) d \xi \\
& =\phi_{i j, m}(s) \int_{-\infty}^{\infty} \eta^{s}\left(\xi^{2}+\eta^{2}\right)^{-s} e(m \xi) d \xi \\
& =\phi_{i j, m}(s) \int_{-\infty}^{\infty} \eta^{s}\left(\xi^{2}+\eta^{2}\right)^{-s} e(-m \xi) d \xi \\
& =\phi_{i j, m}(s) \cdot B_{s}(z, m) \cdot e(m \xi), \quad(m \neq 0),
\end{aligned}
$$

and

$$
a_{i j, 0}(\eta, s)=\delta_{i j} \eta^{s}+\phi_{i j}(s) \eta^{1-s}, \quad\left(\delta_{i j}: \text { Kronecker's delta }\right)
$$

Here,

$$
\phi_{i j, m}(s)=\sum_{m}|c|^{-2 s}\left(\sum_{d} e\left(-\frac{m d}{c}\right)\right), \quad\left(c>0, d(\bmod c),\left(\begin{array}{ll}
* & * \\
c & d
\end{array}\right) \in \sigma_{i}^{-1} \Gamma \sigma_{j}\right),
$$

and

$$
\phi_{i j}(s)=\phi_{i j, 0}(s) B_{s}(z, 0) \eta^{s-1} .
$$

Therefore, we have the expansion of the Eisenstein series $E_{i}(z, s)$ at the cusp $\kappa_{j}$ by "Whittaker function" $B_{s}(z, u)$ :

$$
E_{i}\left(\sigma_{j} z, s\right)=\delta_{i j} \eta^{s}+\sum_{m \in Z} \phi_{i j, m}(s) B_{s}(z, m) .
$$

The constant term matrix $\Phi(s)=\left(\phi_{i j}(s)\right)$ is a symmetric matrix. Furthermore, $\Phi(s)$ is meromorphic on the whole $s$-plane and satisfies the functional equation $\Phi(s) \Phi(1-s)=I$, where $I$ is the identity matrix. 
Each $E_{i}(z, s)$ has a meromorphic continuation to the whole $s$-plane, and the column vector $\mathscr{E}(z, s)={ }^{t}\left(E_{1}(z, s), \cdots, E_{h}(z, s)\right)$ satisfies the functional equation

$$
\mathscr{E}(z, s)=\Phi(s) \mathscr{E}(z, 1-s)
$$

Therefore, each $\phi_{i j, m}(s)$ has a meromorphic continuation to the whole $s$-plane, and the both sides of (2.2) has a meaning as meromorphic functions on the whole $s$-plane.

Now, we prove the following theorem.

TheORem 2. For a discontinuous group $\Gamma$ of finite type with inequivalent cusps $\kappa_{j}(j=1, \cdots, h)$, let $\phi_{i j, m}(s)(i, j=1, \cdots, h, m \in Z)$ be the Dirichlet series defined by:

$$
\phi_{i j, m}(s)=\sum_{c}|c|^{-2 s}\left(\sum_{d} e\left(-\frac{m d}{c}\right)\right), \quad\left(c>0, d(\bmod c),\left(\begin{array}{ll}
* & * \\
c & d
\end{array}\right) \in \sigma_{i}^{-1} \Gamma \sigma_{j}\right),
$$

where $\sigma_{i} \in S L(2, R)$ are as above. Further, let $r_{s}$ be the "Weil type representation" of $S L(2, R)$ in the space $\mathscr{B}_{s}$, defined in Theorem 1.

Then, for any $\sigma \in \Gamma^{(j)}=\sigma_{j}^{-1} \Gamma \sigma_{j}$, we have

$$
\delta_{i j} \cdot \Phi(\infty)+\sum_{m \in Z} \phi_{i j, m}(s) \Phi(m)=\delta_{i j} \cdot\left(r_{s}(\sigma) \Phi\right)(\infty)+\sum_{m \in Z} \phi_{i j, m}(s)\left(r_{s}(\sigma) \Phi\right)(m),
$$

where $\Phi(u) \in \mathscr{B}_{s}^{0}\left(\right.$ i.e., $\Phi(u)=B_{s}(f, u)$ for $\left.f(z) \in C_{0}(H)\right)$ and

$$
\Phi(\infty)=\int_{H} f(z) \eta^{s} d z, \quad\left(r_{s}(\sigma) \Phi\right)(\infty)=\int_{H} f(z) \eta\left(\sigma^{-1} z\right)^{s} d z
$$

In particular, for $\sigma \in \Gamma^{(j)}=\sigma_{j}^{-1} \Gamma \sigma_{j}$, we have

$$
\delta_{i j} \eta^{s}+\sum_{m \in Z} \phi_{i j, m}(s) B_{s}(z, m)=\delta_{i j} \eta\left(\sigma^{-1} z\right)^{s}+\sum_{m \in Z} \phi_{i j, m}(s) r_{s}(\sigma) B_{s}(z, m) .
$$

Proof. By the fact that $E_{i}(\sigma z, s)=E_{i}(z, s)$ for any $\sigma \in \Gamma$, it follows that $E_{i}\left(\sigma \sigma_{j} z, s\right)=E_{i}\left(\sigma_{j} z, s\right)$ for any $\sigma \in \Gamma$. Since there exist a $\sigma^{\prime} \in \Gamma^{(j)}$ $=\sigma_{j}^{-1} \Gamma \sigma_{j}$ such that $\sigma \sigma_{j}=\sigma_{j} \sigma^{\prime}$ for any $\sigma \in \Gamma$, we conclude that $E_{i}\left(\sigma_{j} \sigma z, s\right)$ $=E_{i}\left(\sigma_{j} z, s\right)$ for any $\sigma \in \Gamma^{(f)}$. Taking into account the expansion (2.2) of $E_{i}\left(\sigma_{j} z, s\right)$, we have

$$
\delta_{i j} \eta^{s}+\sum_{m \in Z} \phi_{i j, m}(s) B_{s}(z, m)=\delta_{i j} \eta\left(\sigma^{-1} z\right)^{s}+\sum_{m \in Z} \phi_{i j, m}(s) B_{s}\left(\sigma^{-1} z, m\right)
$$

for any $\sigma \in \Gamma^{(j)}$.

By means of Theorem 1, we can rewrite this formula in the form: 


$$
\delta_{i j} \eta^{s}+\sum_{m \in Z} \phi_{i j, m}(s) B_{s}(z, m)=\delta_{i j} \eta\left(\sigma^{-1} z\right)^{s}+\sum_{m \in Z} \phi_{i j, m}(s) r_{s}(\sigma) B_{s}(z, m)
$$

for any $\sigma \in \Gamma^{(j)}$.

Multiply by $f(z) \in C_{0}(H)$ on the both sides of this formula, and integrate it on $H$ with respect to $d z$, then we obtain the assertion of Theorem 2.

Corollary. Assume that the Eisenstein series $E_{i}(z, s)$ has a pole at $s=s_{0}$. Set $a_{i j, m}$ the residue of $\phi_{i j, m}(s)$ at the pole $s_{0}$.

Then, for any $\sigma \in \Gamma^{(j)}$, we have

$$
\sum_{m \in \boldsymbol{Z}} a_{i j, m} \Phi(m)=\sum_{m \in \boldsymbol{Z}} a_{i j, m}\left(r_{s 0}(\sigma) \Phi\right)(m)
$$

and

$$
\sum_{m \in Z} a_{i j, m} B_{s_{0}}(z, m)=\sum_{m \in Z} a_{i j, m} r_{s_{0}}(\sigma) B_{s_{0}}(z, m),
$$

where $\Phi(u) \in \mathscr{B}_{s_{0}}^{0}$.

Remark 1. We can define "Weil type representations" and "Whittaker functions" for $S L(2, C)$, similarly to the case of $S L(2, R)$. Then we obtain similar results to those of $\S 2$.

Remark 2. In [6], T. Kubota defined generalized theta series by the residues of a kind of Eisenstein series for $S L(2, C)$, and gave a generalized Poisson summation formula associated with the theta series for the special element $\sigma=\left(\begin{array}{ll}-1 & 1\end{array}\right)$. Applying the arguments in Remark 1 to Eisenstein series, which T. Kubota discussed, and its residues, we can get the generalized Poisson summation formula for any element of the corresponding discontinuous group.

\section{§ 3. Generalized Poisson summation formulae for holomorphic forms}

Let $\Gamma$ be a discontinuous group of finite type such that $\infty$ is a cusp and $\Gamma_{\infty}=\left\{\left(\begin{array}{ll}1 & \beta \\ 0 & 1\end{array}\right): \beta \in Z\right\}$. By a similar way to $\S 2$, we can define "Eisenstein series" $\quad E(z, s)_{k}=\sum_{\sigma}|\gamma z+\delta|^{-2 s+k}(\gamma z+\delta)^{-k}, \quad\left(\sigma=\left(\begin{array}{ll}* & * \\ \gamma & \delta\end{array}\right)\right.$ $\left.\in \Gamma_{\infty} \backslash \Gamma\right)$, and obtain "generalized Poisson summation formula" for $E(z, s)_{k}$. But, it is more interesting and important for us to investigate "generalized 
Poisson summation formulae" for automorphic forms of holomorphic type with respect to $\Gamma$.

To carry this out, we start with considering "Weil type representation" and "Whittaker function" for the case where $s=k / 2$ ( $k$ : positive integer). For the sake of brevity, we write $B_{k}(z, u)$ (resp. $r_{k}, K_{k}(\sigma: u, v)$ ) instead of $B_{k / 2, k}(z, u)$ (resp. $r_{k / 2, k}, K_{k / 2, k}(\sigma: u, v)$ ).

We know that $B_{k}(z, u) \in \tilde{F}_{\bar{k}}$ and the representation $\left(r_{k}, \tilde{F}_{\bar{k}}^{-}\right)$is unitary and irreducible (the scalar product in $\tilde{F}_{\bar{k}}$ is given by $\left.(1.12)^{\prime}\right)$. So, we have

$$
B_{k}(z, u)=0 \quad \text { if } u>0
$$

and

$$
K_{k}(\sigma: u, v)=0 \quad \text { if } u v<0,
$$

where $K_{k}(\sigma: u, v)$ is the integral kernel for $r_{k}$.

"Whittaker function" $B_{k}(z, u)$ is given by

$$
B_{1}(z, u)= \begin{cases}-2 \pi \sqrt{-1} & \text { if } u=0 \\ -2 \pi \sqrt{-1} \cdot e(|u| z) & \text { if } u<0\end{cases}
$$

and

$$
B_{k}(z, u)=\left\{\begin{array}{ll}
0 & \text { if } u=0 \\
\frac{(-2 \pi \sqrt{-1})^{k}}{(k-1) !}|u|^{k-1} e(|u| z) & \text { if } u<0
\end{array} \quad(k>1)\right.
$$

The operator $r_{k}(\sigma), \sigma=\left(\begin{array}{ll}\alpha & \beta \\ \gamma & \delta\end{array}\right) \in S L(2, R)$ on the space $\tilde{F}_{k}^{-}$, is given as follows: if $\gamma=0$, then

$$
\begin{aligned}
\left(r_{s}(\sigma) \Phi\right)(u) & =\operatorname{sgn}^{k} \delta|\delta|^{-k+2} e(\delta \beta u) \Phi\left(\delta^{2} u\right) \\
& =\delta^{-k+2} e(\delta \beta u) \Phi\left(\delta^{2} u\right), \quad \Phi(u) \in \tilde{F}_{k}^{-}
\end{aligned}
$$

if $\gamma \neq 0$, then

$$
\begin{aligned}
\left(r_{s}(\sigma) \Phi\right)(u)= & 2 \pi \cdot \operatorname{sgn}^{k} \gamma \cdot e(k / 4)|\gamma|^{-1} \int_{-\infty}^{0} e\left(\frac{\delta u+\alpha v}{\gamma}\right)\left|\frac{u}{v}\right|^{(k-1) / 2} \\
& \cdot J_{k-1}\left(4 \pi \frac{\sqrt{u v}}{|\gamma|}\right) \Phi(v) d v
\end{aligned}
$$

where $u \leqq 0, \Phi(u) \in \tilde{F}_{\bar{k}}$ and $J_{k-1}(x)=\left(H_{k-1}^{(1)}(x)+H_{k-1}^{(2)}(x)\right) / 2$. 
The intertwining formula is given by

$$
r_{k}(\sigma) B_{k}(z, u)=B_{k}\left(\sigma^{-1} z, u\right) j_{k}\left(\sigma^{-1}, z\right), \quad \sigma \in S L(2, R) .
$$

A holomorphic function $f(z)$ on $H$ is called "an automorphic form of holomorphic $k$-type with respect to $\Gamma$ ", if the followings are satisfied:

(1) $f(\sigma z) j_{k}(\sigma, z)=f(z)$ for any $\sigma \in \Gamma$,

(2) $f(z)$ is holomorphic at every cusp of $\Gamma$.

In explanation of the last condition, fix an element $\sigma_{i} \in S L(2, R)$ for every cusp $\kappa_{i}$ such that $\sigma_{i} \infty=\kappa_{i}, \sigma_{i}^{-1} \Gamma_{i} \sigma_{i}=\left\{\left(\begin{array}{ll}1 & \beta \\ 0 & 1\end{array}\right): \beta \in Z\right\}$, then (2) says that $f_{i}(z)$ $=f\left(\sigma_{i} z\right) j_{k}\left(\sigma_{i}, z\right)$ has the Fourier expansion:

$$
f_{i}(z)=a_{i, 0}+\sum_{n \geq 1} a_{i, n} e(n z) .
$$

The coefficients $a_{i, n}$ are called the Fourier coefficients of $f(z)$ at the cusp $\kappa_{i}$.

We define "modified coefficients" $b_{i, n}$ of $f(z)$ at the cusp $\kappa_{i}$ by

$$
b_{i, n}=\left\{\begin{array}{ll}
0 & \text { if } n>0, \\
\frac{-a_{i,-n}}{2 \pi \sqrt{-1}} & \text { if } n \leqq 0,
\end{array} \quad(k=1),\right.
$$

and

$$
b_{i, n}=\left\{\begin{array}{ll}
0 & \text { if } n \geqq 0, \\
\frac{(k-1) !}{(-2 \pi \sqrt{-1})^{k}} \cdot|n|^{-k+1} \cdot a_{i,-n} & \text { if } n<0, \\
a_{i, 0} & \text { if } n=\infty
\end{array} \quad(k>1) .\right.
$$

Then we have the expansion of $f_{i}(z)$ by means of "Whittaker functions":

$$
f_{i}(z)= \begin{cases}b_{i, \infty}+\sum_{n \in Z} b_{i, n} B_{k}(z, n) & \text { if } k>1 \\ \sum_{n \in Z} b_{i, n} B_{1}(z, n) & \text { if } k=1\end{cases}
$$

Since $f_{i}\left(\sigma^{-1} z\right) j_{k}\left(\sigma^{-1}, z\right)=f_{i}(z)$ for any $\sigma \in \Gamma^{(i)}=\sigma_{i} \Gamma \sigma_{i}^{-1}$, we get

$$
\begin{aligned}
b_{i, \infty}+\sum_{n \in \boldsymbol{Z}} b_{i, n} B_{k}(z, n) & =b_{i, \infty} j_{k}\left(\sigma^{-1}, z\right)+\sum_{n \in \boldsymbol{Z}} b_{i, n} B_{k}\left(\sigma^{-1} z, n\right) j_{k}\left(\sigma^{-1}, z\right) \\
& =b_{i, \infty} j_{k}\left(\sigma^{-1}, z\right)+\sum_{n \in \boldsymbol{Z}} b_{i, n} r_{k}(\sigma) B_{k}(z, n)
\end{aligned}
$$

for $k>1$, and 


$$
\begin{aligned}
\sum_{n \in Z} b_{i, n} B_{1}(z, n) & =\sum_{n \in Z} b_{i, n} B_{1}\left(\sigma^{-1} z, n\right) j_{1}\left(\sigma^{-1}, z\right) \\
& =\sum_{n \in \boldsymbol{Z}} b_{i, n} r_{1}(\sigma) B_{1}(z, n)
\end{aligned}
$$

for $k=1$.

THEOREM 3. Let $b_{i, n}(i=1, \cdots, h, n \in Z \cup\{\infty\})$ be "modified coefficients" of an automorphic form $f(z)$ of holomorphic k-type with respect to $\Gamma$, at the cusp $\kappa_{i}$, and $\left(r_{k}, \tilde{F}_{\bar{k}}^{-}\right)$be the "Weil type representation" of $S L(2, R)$, defined by (3.2) and (3.2)'.

Then we have; for any $\sigma \in \Gamma^{(i)}$,

$$
\sum_{n \in Z} b_{i, n} \Phi(n)=\sum_{n \in Z} b_{i, n}\left(r_{1}(\sigma) \Phi\right)(n), \quad(k=1),
$$

and

$$
\begin{array}{r}
b_{i, \infty} \Phi(\infty)+\sum_{n \in Z} b_{i, n} \Phi(n)=b_{i, \infty}\left(r_{k}(\sigma) \Phi\right)(\infty)+\sum_{n \in Z} b_{i, n}\left(r_{k}(\sigma) \Phi\right)(n), \\
(k>1),
\end{array}
$$

where $\Phi(u) \in\left(\tilde{F}_{\bar{k}}\right)^{0}\left(i . e ., \Phi(u)=B_{k}(g, u)\right.$ for $\left.g \in C_{0}(H)\right)$ and

$$
\Phi(\infty)=\int_{H} g(z) d z, \quad\left(r_{k}(\sigma) \Phi\right)(\infty)=\int_{H} g(z) j_{k}\left(\sigma^{-1}, z\right) d z
$$

In particular,

$$
\begin{gathered}
\sum_{n \in Z} b_{i, n} B_{1}(z, n)=\sum_{n \in Z} b_{i, n} r_{k}(\sigma) B_{1}(z, n), \quad(k=1), \\
b_{i, \infty}+\sum_{n \in Z} b_{i, n} B_{k}(z, n)=b_{i, \infty} j_{k}\left(\sigma^{-1}, z\right)+\sum_{n \in Z} b_{i, n} r_{k}(\sigma) B_{k}(z, n), \quad(k>1),
\end{gathered}
$$

for any $\sigma \in \Gamma^{(i)}$.

Conversely, for any sequence $\left\{b_{n}\right\},(n \in Z \cup\{\infty\})$, for which the formula (3.5) or (3.5)' is valid for any $\sigma \in \Gamma^{(i)}, b_{n}$ are "modified coefficients" of an automorphic form of holomorphic k-type with respect to $\Gamma$, at the cusp $\kappa_{i}$.

Remark. It is not difficult to generalize the above results to the case of non-holomorphic forms, which satisfy a kind of differential equation. More precisely, we can expand non-holomorphic forms by means of "Whittaker functions".

\section{$\S 4$. Covering groups of $S L(2, R)$ and Weil representation}

For any positive integer $q$, let $G^{(q)}$ denote the set of $\tilde{\sigma}=(\sigma, j(\tilde{\sigma}, z))$ 
formed by an element $\sigma=\left(\begin{array}{ll}\alpha & \beta \\ \gamma & \delta\end{array}\right) \in S L(2, R)$ and a holomorphic function $j(\tilde{\sigma}, z)$ on the upper half plane $H$ such that $j(\tilde{\sigma}, z)^{q}=\gamma z+\delta$. Defining a law of multiplication in $G^{(q)}$ by

$$
(\tau, j(\tilde{\tau}, z)) \cdot(\sigma, j(\tilde{\sigma}, z))=(\tau \sigma, j(\tilde{\tau}, \sigma z) j(\tilde{\sigma}, z)),
$$

we can make $G^{(q)}$ a group with the unit element $\left(\left(\begin{array}{ll}1 & 0 \\ 0 & 1\end{array}\right), 1\right)$. It is clear that $G^{(q)}$ is a $q$-folded covering group of $S L(2, R)$.

For $\tilde{\sigma}=(\sigma, j(\tilde{\sigma}, z)) \in G^{(q)}$, set

$$
\phi_{s, p}(\tilde{\sigma}, z)=j(\tilde{\sigma}, z)^{-p} \eta(\sigma z)^{s-(p / 2 q)},
$$

where $s$ is a complex variable and $p$ is an integer which is relatively prime to $q$ and $0<p<q$. Then we have, for any $\tilde{\tau}, \tilde{\sigma} \in G^{(q)}$,

$$
\phi_{s, p}(\tilde{\tau} \tilde{\sigma}, z)=\phi_{s, p}(\tilde{\tau}, \sigma z) \cdot j(\tilde{\sigma}, z)^{-p} .
$$

For any $z \in C$, we define ${ }^{q} \sqrt{z}=z^{1 / q}$ so that $-\pi / q<\arg { }^{q} \sqrt{z} \leqq \pi / q$, then it follows that, for $z_{2} / z_{1} \in H, z_{1}, z_{2} \in C-R$,

$$
\left(z_{2} / z_{1}\right)^{1 / q}\left(z_{1}\right)^{1 / q}= \begin{cases}e(1 / q) \cdot\left(z_{2}\right)^{1 / q}, & \text { if } z_{1} \in H \text { and } \bar{z}_{2} \in H, \\ \left(z_{2}\right)^{1 / q}, & \text { otherwise },\end{cases}
$$

and, for $z \in H, c \in R$,

$$
(c z)^{1 / q}= \begin{cases}|c|^{1 / q} z^{1 / q} & \text { if } c>0, \\ c(-1 / 2 q)|c|^{1 / q} z^{1 / q} & \text { if } c<0 .\end{cases}
$$

For any $\tilde{\sigma}=(\sigma, j(\tilde{\sigma}, z)) \in G^{(q)}, \sigma=\left(\begin{array}{ll}\alpha & \beta \\ \gamma & \delta\end{array}\right)$, there exist unique $q$-th root $\varepsilon(\tilde{\sigma})$ of unity such that $j(\tilde{\sigma}, z)=\varepsilon(\tilde{\sigma})(\gamma z+\delta)^{1 / q}$.

Put

$$
\phi_{s, p}(z, x)=(z-x)^{-p / q}|z-x|^{-2 s+(p / q)} \eta^{s-(p / 2 q)}, \quad z \in H, x \in R,
$$

then we have

$$
\phi_{s, p}(z, x)=\phi_{s, p}(\tilde{\tau}, z)
$$

for $\tilde{\tau}=(\tau, j(\tilde{\tau}, z)) \in G^{(q)}, \tau=\left(\begin{array}{rr}* & * \\ 1 & -x\end{array}\right), j(\tilde{\tau}, z)=(z-x)^{1 / q}$.

For $\tilde{\sigma}=(\sigma, j(\tilde{\sigma}, z)) \in G^{(q)}, \sigma=\left(\begin{array}{ll}\alpha & \beta \\ \gamma & \delta\end{array}\right)$, we put 


$$
j(\tilde{\sigma}, x)= \begin{cases}\varepsilon(\tilde{\sigma})^{-1}|\gamma x+\delta|^{1 / q} & \text { if } \gamma x+\delta>0, \gamma \neq 0, \\ \varepsilon(\tilde{\sigma})^{-1} e(\operatorname{sgn} \gamma / 2 q)|\gamma x+\delta|^{1 / q} & \text { if } \gamma x+\delta<0, \gamma \neq 0, \\ \varepsilon(\tilde{\sigma})^{-1} \delta^{1 / q} & \text { if } \gamma=0\end{cases}
$$

Then, for the above $\tilde{\tau} \in G^{(q)}$ and for any $\tilde{\sigma} \in G^{(q)}, \sigma=\left(\begin{array}{ll}\alpha & \beta \\ \gamma & \delta\end{array}\right)$, we can see that

$$
\phi_{s, p}\left(\tilde{\tau} \tilde{\sigma}^{-1}, z\right)=j(\tilde{\sigma}, x)^{-p}|\gamma x+\delta|^{-2 s+(p / q)} \phi_{s, p}\left(z, \frac{\alpha x+\beta}{\gamma x+\delta}\right),
$$

because (4.3) and (4.4) imply that

$$
\left(\frac{\delta z-\beta}{-\gamma z+\alpha}-x\right)^{-1 / q}(-\gamma z+\sigma)^{-1 / q}=\left\{\begin{array}{r}
|\gamma x+\delta|^{-1 / q}\left(z-\frac{\alpha x+\beta}{\gamma x+\delta}\right)^{-1 / q} \\
\text { if } \gamma x+\delta>0, \\
e(-1 / 2 q)|\gamma x+\delta|^{-1 / q}\left(z-\frac{\alpha x+\beta}{\gamma x+\delta}\right)^{-1 / q} \\
\text { if } \gamma x+\delta<0, \gamma>0, \\
e(1 / 2 q)|\gamma x+\delta|^{-1 / q}\left(z-\frac{\alpha x+\beta}{\gamma x+\delta}\right)^{-1 / q} \\
\text { if } \gamma x+\delta<0, \gamma<0 .
\end{array}\right.
$$

Let $\mathscr{A}_{s, p}^{(q)}$ be the vector space generated by functions $\phi_{s, p}(z, x)$ on $x$, where the topology in $\mathscr{A}_{s, p}^{(q)}$ is defined similarly to that of $\mathscr{A}_{s, k}$ in $\S 1$. The representation $\pi_{s, p}$ of $G^{(q)}$ in the space $\mathscr{A}_{s, p}^{(q)}$ is defined by

$$
\pi_{s, p}(\tilde{\sigma}) \phi(x)=\phi\left(\frac{\alpha x+\beta}{\gamma x+\delta}\right) j(\tilde{\sigma}, x)^{-p}|\gamma x+\delta|^{-2 s+(p / q)}, \quad \phi(x) \in \mathscr{A}_{s, p}^{(q)},
$$

for $\tilde{\sigma}=(\sigma, j(\tilde{\sigma}, z)) \in G^{(q)}, \sigma=\left(\begin{array}{ll}\alpha & \beta \\ \gamma & \delta\end{array}\right) \in S L(2, R)$.

By means of (4.2) and (4.6), we get the following intertwining formula:

$$
\pi_{s, p}(\tilde{\sigma}) \phi_{s, p}(z, x)=\phi_{s, p}\left(\sigma^{-1} z, x\right) \cdot j\left(\tilde{\sigma}^{-1}, z\right)^{-p},
$$

for any $\tilde{\sigma} \in G^{(q)}$.

For this representation $\pi_{s, p}$, we can obtain similar results to those for $\pi_{s, k}$ in $\S 1$, but here we are interested in the case when $s=p / 2 q$. We write $\pi_{p / q}$ (resp. $\mathscr{A}_{p / q}$ ) instead of $\pi_{p / 2 q, p}$ (resp. $\mathscr{A}_{p / 2 q, p)}^{(q)}$ ). The representation $\left(\pi_{p / q}, \mathscr{A}_{p / q}\right)$ is irreducible and unitary with the scalar product 


$$
\begin{array}{r}
(\phi, \psi)_{p / q}=\int_{-\infty}^{\infty} \int_{-\infty}^{\infty}\left(x_{1}-x-\sqrt{-1} \cdot 0\right)^{(p / q)-2} \phi\left(x_{1}\right) \cdot \bar{\psi}(x) d x_{1} d x, \\
\phi, \psi \in \mathscr{A}_{p / q} .
\end{array}
$$

Denote by $\mathscr{B}_{s, p}^{(q)}$ the vector space generated by "Whittaker function" $B_{s, p}(z, u)$ on $u \in R$, where

$$
\begin{aligned}
B_{s, p}(z, u) & =\int_{-\infty}^{\infty} \phi_{s, p}(z, x) e(-u x) d x \\
& =\int_{-\infty}^{\infty} \eta^{s-(p / 2 q)}(z-x)^{-s-(p / 2 q)}(\bar{z}-x)^{-s+(p / 2 q)} e(-u x) d x
\end{aligned}
$$

This integral is expressed by the usual Whittaker function $W_{\kappa, \mu}$ :

$$
\begin{aligned}
& B_{s, p}(z, u) \\
& =\left\{\begin{array}{cc}
2 \pi(\sqrt{-1})^{-p / q} \frac{\Gamma(2 s-1)}{\Gamma(s+(p / 2 q)) \Gamma(s-(p / 2 q))}(2 \eta)^{1-2 s} \eta^{s-(p / 2 q)} & \text { if } u=0, \\
\frac{\pi^{s}(\sqrt{-1})^{-p / q}}{\Gamma(s-(p / 2 q))}|u|^{s-1} \eta^{-p / 2 q} W_{-p / 2 q, s-(1 / 2)}(4 \pi|u| \eta) e(-u \xi) & \text { if } u>0, \\
\frac{\pi^{s}(\sqrt{-1})^{-p / q}}{\Gamma(s+(p / 2 q))}|u|^{s-1} \eta^{-p / 2 q} W_{p / 2 q, s-(1 / 2)}(4 \pi|u| \eta) e(-u \xi) & \text { if } u<0, \\
(z=\xi+i \eta \in H) . &
\end{array}\right.
\end{aligned}
$$

By means of Fourier transformation and by similar arguments to $\S 1$, we get another realization $\left(r_{s, p}, \mathscr{B}_{s, p}^{(q)}\right)$ of the representation $\pi_{s, p}$ of $G^{(q)}$.

If, namely, $\tilde{\sigma}=(\sigma, j(\tilde{\sigma}, z)) \in G^{(q)}, \sigma=\left(\begin{array}{ll}\alpha & \beta \\ \gamma & \delta\end{array}\right) \in S L(2, \boldsymbol{R}), \gamma=0$, then

$$
\left(r_{s, p}(\tilde{\sigma}) \Phi\right)(u)=\varepsilon(\tilde{\sigma})^{p}(\operatorname{sgn} \delta)^{-p / q}|\delta|^{-2 s+2} e(\delta \beta u) \Phi\left(\delta^{2} u\right), \quad \Phi(u) \in \mathscr{B}_{s, p}^{(q)}
$$

If $\tilde{\sigma}=(\sigma, j(\tilde{\sigma}, z)) \in G^{(q)}, \sigma=\left(\begin{array}{cc}\alpha & \beta \\ \gamma & \delta\end{array}\right) \in S L(2, R), \gamma \neq 0$, then

$$
\left(r_{s, p}(\tilde{\sigma}) \Phi\right)(u)=\int_{-\infty}^{\infty} K_{s, p}(\tilde{\sigma}: u, v) \Phi(v) d v, \quad \Phi(u) \in \mathscr{B}_{s, p}^{(q)},
$$

where the integral kernel $K_{s, p}(\tilde{\sigma}: u, v)$ is given by the followings: in the case that $u v>0$,

$$
\begin{aligned}
K_{s, p}(\tilde{\sigma}: u, v)= & \varepsilon(\tilde{\sigma})^{p} \sqrt{-1} \cdot|\gamma|^{-1} e\left(\frac{\delta u+\alpha v}{\gamma}\right)\left|\frac{u}{v}\right|^{s-(1 / 2)} \\
& \cdot\left[e(-p(\operatorname{sgn} \gamma+\operatorname{sgn} u) / 4 q) e((2 s-1) / 4) H_{2 s-1}^{(1)}\left(4 \pi \frac{\sqrt{u v}}{|\gamma|}\right)\right.
\end{aligned}
$$




$$
\left.-e(-p(\operatorname{sgn} \gamma-\operatorname{sgn} u) / 4 q) e((1-2 s) / 4) H_{2 s-1}^{(2)}\left(4 \pi \frac{\sqrt{u v}}{|\gamma|}\right)\right]
$$

in the case of that $u v<0$,

$$
\begin{aligned}
K_{s, p}(\tilde{\sigma}: u, v)= & \varepsilon(\tilde{\sigma})^{p} 2|\gamma|^{-1} e\left(\frac{\delta u+\alpha v}{\gamma}\right)\left|\frac{u}{v}\right|^{s-(1 / 2)} \\
& \times[e(-p(\operatorname{sgn} \gamma+\operatorname{sgn} u) / 4 q) e((2 s-1) / 4) \\
& +e(-p(\operatorname{sgn} \gamma-\operatorname{sgn} u) / 4 q) e((1-2 s) / 4)] K_{2 s-1}\left(4 \pi \frac{\sqrt{|u v|}}{|\gamma|}\right) .
\end{aligned}
$$

Furthermore, we have the following intertwining formula:

$$
r_{s, p}(\tilde{\sigma}) \cdot B_{s, p}(z, u)=B_{s, p}\left(\tilde{\sigma}^{-1} z, u\right) \cdot j\left(\tilde{\sigma}^{-1}, z\right)
$$

for any $\tilde{\sigma} \in G^{(q)}$.

Now, we investigate the case where $s=p / 2 q$. We write $r_{p / q}$ (resp. $\left.B_{p / q}(z, u), \mathscr{B}_{p / q}\right)$ instead of $r_{p / 2 q, p}\left(\operatorname{resp} . B_{p / 2 q, p}(z, u), \mathscr{B}_{p / 2 q, p}^{(q)}\right)$. We see that

$$
B_{p / q}(z, u)= \begin{cases}0 & \text { if } u \leqq 0, \\ \left(\frac{2 \pi}{\sqrt{-1}}\right)^{p / q} \Gamma(p / q)^{-1} u^{(p / q)-1} e(-z u) & \text { if } u<0 .\end{cases}
$$

The operator $r_{p / q}(\tilde{\sigma})$ for $\tilde{\sigma}=(\sigma, j(\tilde{\sigma}, z)) \in G^{(q)}, \sigma=\left(\begin{array}{ll}\alpha & \beta \\ \gamma & \delta\end{array}\right), \gamma=0$, is given by

$$
\left(r_{p / q}(\tilde{\sigma}) \Phi\right)(u)=\varepsilon(\tilde{\sigma})^{p} \delta^{2-(p / q)} e(\delta \beta u) \Phi\left(\delta^{2} u\right), \quad \Phi(u) \in \mathscr{B}_{p / q} .
$$

The operator $r_{p / q}(\tilde{\sigma})$ for $\tilde{\sigma}=(\sigma, j(\tilde{\sigma}, z)) \in G^{(q)} \sigma=\left(\begin{array}{ll}\alpha & \beta \\ \gamma & \delta\end{array}\right), \gamma \neq 0$, is given by

$$
\left(r_{p / q}(\tilde{\sigma}) \Phi\right)(u)=\left\{\begin{array}{ll}
0 & \text { if } u \geqq 0, \\
\int_{-\infty}^{0} K_{p / q}(\tilde{\sigma}: u, v) \Phi(v) d v, & \text { if } u<0,
\end{array} \Phi(u) \in \mathscr{B}_{p / q},\right.
$$

where

$$
\begin{aligned}
K_{p / q}(\tilde{\sigma}: u, v)= & \varepsilon(\tilde{\sigma})^{p} 2 \pi|\gamma|^{-1} e\left(\frac{\delta u+\alpha v}{\gamma}\right)\left|\frac{u}{v}\right|^{(p-q) / 2 q} \\
& \cdot e(-\operatorname{sgn} \gamma \cdot p / 4 q) J_{(p / q)-1}\left(4 \pi \frac{\sqrt{u v}}{|\gamma|}\right) .
\end{aligned}
$$

The representation $\left(r_{p / q}, \mathscr{B}_{p / q}\right)$ is irreducible and unitary with the scalar product 


$$
(\Phi, \Psi)_{p / q}=\int_{-\infty}^{0}|u|^{1-(p / q)} \Phi(u) \bar{\Psi}(u) d u, \quad \Phi, \Psi \in \mathscr{B}_{p / q}
$$

Further, considering the special case where $p / q=1 / 2$, we can see that

$$
\begin{aligned}
K_{1 / 2}(\tilde{\sigma}: u, v) & =\varepsilon(\tilde{\sigma}) \cdot 2 \pi|\gamma|^{-1} e(-\operatorname{sgn} \gamma / 8) e\left(\frac{\delta u+\alpha v}{\gamma}\right)\left|\frac{u}{v}\right|^{-1 / 4} J_{-1 / 2}\left(4 \pi \frac{\sqrt{u v}}{|\gamma|}\right) \\
& =\sqrt{2} \varepsilon(\tilde{\sigma}) e(-\operatorname{sgn} \gamma / 8)|\gamma|^{-1 / 2} e\left(\frac{\delta u+\alpha v}{\gamma}\right)|u|^{-1 / 2} \cos \left(4 \pi \frac{\sqrt{u v}}{\gamma}\right) .
\end{aligned}
$$

We modify the representation $\left(r_{1 / 2}, \mathscr{B}_{1 / 2}\right)$ in the following way. Let $\mathscr{B}$ be the function space consisting of $\Phi(t)=|t| \Phi_{1}\left(-t^{2} / 2\right)$ for any $\Phi_{1}(u) \in \mathscr{B}_{1 / 2}$, and let $r$ be the representation of $G^{(2)}$ in the space $\mathscr{B}$ defined by

$$
(r(\tilde{\sigma}) \Phi)(t)=|t|\left(r_{1 / 2}(\tilde{\sigma}) \Phi_{1}\right)\left(-t^{2} / 2\right), \quad \Phi(t)=|t| \Phi_{1}\left(-t^{2} / 2\right), \quad \Phi_{1} \in \mathscr{B}_{1 / 2},
$$

for $\tilde{\sigma} \in G^{(2)}$.

Then, the representation $(r, \mathscr{B})$ is irreducible and unitary with the scalar product

$$
\begin{aligned}
(\Phi, \Psi) & =2 \sqrt{2}\left(\Phi_{1}, \Psi_{1}\right)_{1 / 2} \\
& =2 \sqrt{2} \int_{-\infty}^{0}|u|^{1 / 2} \Phi_{1}(u) \bar{\Psi}_{1}(u) d u \\
& =2 \sqrt{2} \int_{0}^{\infty} \frac{|t|}{\sqrt{2}} \Phi_{1}\left(-t^{2} / 2\right) \bar{\Psi}_{1}\left(-t^{2} / 2\right) t d t \\
& =\int_{-\infty}^{\infty} \Phi(t) \cdot \bar{\Psi}(t) d t
\end{aligned}
$$

where $\Phi(t)=|t| \Phi_{1}\left(-t^{2} / 2\right), \Psi(t)=|t| \Psi_{1}\left(-t^{2} / 2\right) \in \mathscr{B}, \Phi_{1}, \Psi_{1} \in \mathscr{B}_{1 / 2}$.

The operation of $r(\tilde{\sigma})$ is given as follows.

If $\tilde{\sigma}=(\sigma, j(\tilde{\sigma}, z)), \sigma=\left(\begin{array}{ll}\alpha & \beta \\ \gamma & \delta\end{array}\right) \in S L(2, R), \gamma=0$, then

$$
\begin{aligned}
(r(\tilde{\sigma}) \Phi)(t) & =\varepsilon(\tilde{\sigma}) \delta^{2-(1 / 2)} e\left(-\delta \beta t^{2} / 2\right) \Phi_{1}\left(-\delta^{2} t^{2} / 2\right)|t| \\
& =\varepsilon(\tilde{\sigma}) \delta^{1 / 2} e\left(-\delta \beta t^{2} / 2\right) \Phi(\delta t) .
\end{aligned}
$$

If $\tilde{\sigma}=(\sigma, j(\tilde{\sigma}, z)), \sigma=\left(\begin{array}{ll}\alpha & \beta \\ \gamma & \delta\end{array}\right) \in S L(2, R), \gamma \neq 0$, then

$$
\begin{aligned}
(r(\tilde{\sigma}) \Phi)(t)= & \sqrt{2} \varepsilon(\tilde{\sigma}) e(-\operatorname{sgn} \gamma / 8)\left|-t^{2} / 2\right|^{-1 / 2}|t| \\
& \cdot \int_{-\infty}^{0} e\left(\frac{\delta\left(-t^{2} / 2\right)+\alpha v}{2 \gamma}\right) \cos \left(4 \pi \frac{\sqrt{\left(-t^{2} / 2\right) v}}{\gamma}\right) \Phi_{1}(v) d v \\
= & 2 \varepsilon(\tilde{\sigma}) e(-\operatorname{sgn} \gamma / 8)|\gamma|^{-1 / 2}
\end{aligned}
$$




$$
\begin{aligned}
& \cdot \int_{0}^{\infty} e\left(-\frac{\delta t^{2}+\alpha\left(t_{1}\right)^{2}}{2 \gamma}\right) \cos \left(2 \pi t \cdot t_{1} / \gamma\right) \Phi_{1}\left(-\left(t_{1}\right)^{2} / 2\right) t_{1} d t_{1} \\
= & \varepsilon(\tilde{\sigma}) e(-\operatorname{sgn} \gamma / 8)|\gamma|^{-1 / 2} \int_{-\infty}^{\infty} e\left(-\frac{\delta t^{2}-2 t_{1} t+\alpha\left(t_{1}\right)^{2}}{2 \gamma}\right) \Phi\left(t_{1}\right) d t_{1} .
\end{aligned}
$$

Therefore, the representation $(r, \mathscr{B})$ is nothing else than the original Weil representation.

Remark 1. The original definition of Weil representation in [11] seems to be somewhat complicated. By means of the above results, we can see the connexion between Weil representation and other representations.

Remark 2. Recently, S. Niwa showed that Weil representation for a non-archimedian local field is obtained by similar arguments to those of $\S 4$.

\section{REFERENCES}

[1] A. Erdélyi, Higher transcendental functions, McGraw-Hill, vol. 1, II, 1953.

[2] I. M. Gel'fand, M. I. Graev and N. Ya. Vilenkin, Generalized functions, 5, Moscow, 1962, Translation: Academic Press, 1966.

[3] I. M. Gel'fand, M. I. Graev and I. I. Pyatetskii-Shapiro, Representation theory and automorphic functions, Moscow, 1966, Translation: Saunder, 1969.

[ 4 ] T. Kubota, Elementary theory of Eisenstein series, Kodansha, 1973.

[ 5 ] — On a generalized Weil type representation, Proc. Int. Symp., Kyoto, 1976.

[6] —-, On an analogy to the Poisson summation formula for generalized Fourier transformation, J. reine angew. Math., 268/269 (1974), 180-189.

[ 7 ] — On a generalized Fourier transformation, J. Fac. of Sci. Univ. of Tokyo, 24, No. 1 (1977), 1-10.

[ 8 ] W. Magnus, F. Oberhettinger and R. P. Soni, Formulas and theorems for the special function of Mathematical Physics, Springer, 1966.

[9] C. L. Siegel, Die Funktionalgleichung einiger Dirichletscher Reihen, Math. Zeitschr., 63 (1956), 363-373.

[10] G. N. Watson, Theory of Bessel functions, Cambridge, 1922.

[11] A. Weil, Sur certains groupes d'opérateurs unitaires, Acta. Math., 111 (1964), 143-211.

Nagoya University

Current address:

Ryukyu University 